Aus der Quarantäne-Abteilung des Allgemeinen Krankenhauses Hamburg-Barmbeck. (Direktor: Prof. Th. Rumpel.)

\title{
Insektensichere Schutzkleidung.')
}

\section{Von A. V. Knack.}

M. H.? Als bei der Mobilmachung unsere Kollegen, dic nach dem Osten ziehen mußten, neben anderem Kriegsgerät eine reichliche Dosis Insektenpulver mitnahmen, dachte wohl kaum einer von uns daran, daß die Frage der Ungezieferbekämpfung eine derart wichtige Rolle in diesen Kriege spielen würde. Und wahrscheinlich hätte man die Ungezieferplage, wie in den früheren Kriegen, so auch in diesem als eine Conditio sine qua non stillschweigend hingenommen, wenn wir nicht durch die Forschung der jüngsten Zeit wüßten, daß eine der am meisten gefürchteten Kriegsseuchen, der Flecktyphus, der in fast allen größeren Kriegen als "Kriegstyphus" zahllose Opfer forderte, durch Ungeziefer, vor allem durch Kleiderläuse, übertragen wird.

Iie Zeit rerbietet es nir, an dieser Stclle auf Einzelheitcn ein\%ugehen, ich vorweise nur auf die umfassende Arbeit von Kirstein in der Fur und Wider der Flecktyphusubertragung durch Läusc eingehend erortert wird.

Unentschieden ist noch die Frage, ob bei den stets mit Bronchiti im Initialstadium behafteten Fleckfieberkranken eine Tropfcheninfektion nöglich ist, wonn man auch nicht gerade das von Petruschky kiilzlich im Sputum von Fleckfiebernden gefundene influenzaühnliche Stäbchrn als Erreger dieser Seuehe ansehen mochtr. Sicherlich ist diese 'l'ropfeheninfektion selten. aber manche Beobachter, wic besonders Brauer und Bujwid, ziehen sie doch inmer noch in Betracht.

Wichtig ist auch die noch mugelostc Frage, ob andcle blutsaugende Insektell, wie Filzlause, Flohe, Wanzen. Mucken. ebenfalls als Uebertrager in Betracht kommon. Das Experintent sprieht bisher noeh dagegen (Ricketts and Wilder), die Bcohachtung mancher Acrzte auf

1) Demonstration in Hanburger Aerztlichen Verein am 1. 6. 1915 
vorgeschohemem Posten (Ninger, Stocklosinski, diujwid) spicht ditt ill.

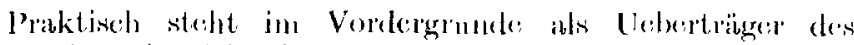
Flecktyphus dic Kleiderlaus, and dic Bekämphnng dicser Senche, ist in erster Linie eine Prophylaxe gegen die Länse.

Leider kam ich vor Ihuen nicht iiber eine Sehutrkleidnm im weitester Sinne des Wortes sprechen, eine Kleidnug. die nuseren Landslenten draußen sicheren Schutz gegen Vor lausming and damit gegen Flecktyphus gewährte, Ich will thnen hier nu' eine Kleidnng demonstrieren, die Arrzten wnd Krankenpflegeru, die zu Flecktyphuspflege herangezogen werden, einen weitgehenden Schuta gegen Ueberkriechen von Länsen und damit gegen Flecktyphnsinfektion gewähron kann.

cerade der Fleektyphus lat wohl, wie keine andere Infektions-

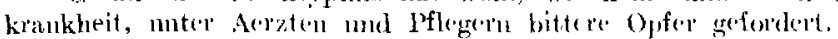

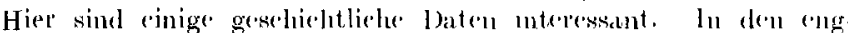

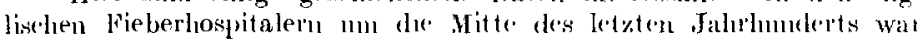

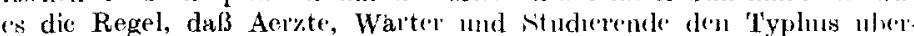

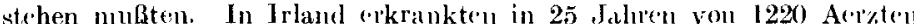
an offentlıchen Austalten 560 m 'lyphus, 132 starben darau. tı de

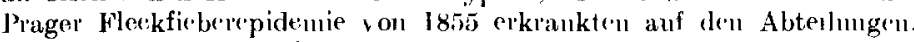
welehe die Kl'ank'n aufuahmen, 20 Ael\%te (davon starbe'n 5) und saint. liehe Wärte'runen, In Kl'iukrıege erkrankten von 840 Wärterı 60; an Typhus, 80 Militariaryte starbe'n 11 diesen Kriege an 'Typhus, Fast stets handeite es sich in diesen Fälon 011 filecktyphos, den man damals noch als eine bosartige Unterart de's Typhons abdominalis ansah

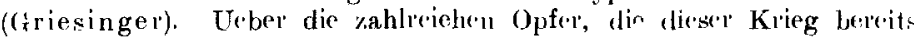
in den Relhen der Unstige'n duech Finektyphusmfektion gefordert hat, sund sie ja alle orientiert.

Nach der allgeme'nen Erfahrung ist e's fur alle die, die mit de' er'sten Aufnahme von Fleekfieberkranke'n und -verdächtige'u in Be'luhrung

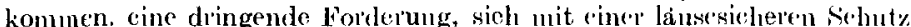
kleidung zu versehen, da es wohl keine unerfrenlicher'e 'Iatsache grlu als durch Unvorsichtigkeit mmotig geopferte Menselienleben.

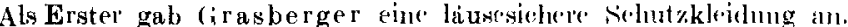
leh zeige Ihnen dic Abbildungen (vgl. W. kl. W' 1915 Ni. (1). Er nimmt

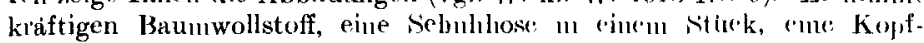
kappenjacke in 'inem stiick, Dic Hose wird voll mutenl, die Jacke uben' den Kopf und Rumpf herabgezog('n, J)ic Verringing ('Ifolgt in der Witte des Rumpfes so, daß \%umachst die Hose iber die Jacke geyogen

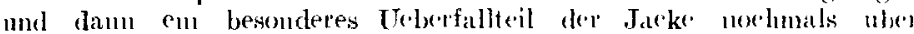

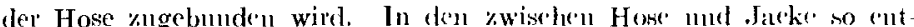

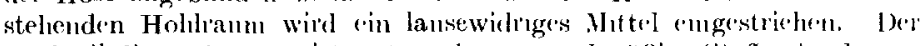

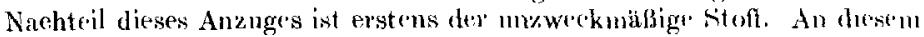

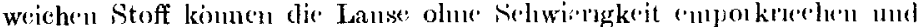

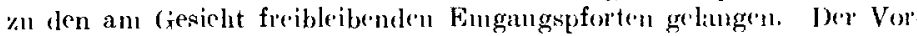

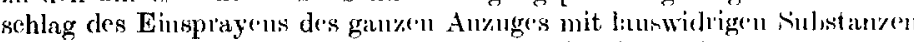
besentigt diessen Nachteil num onm Tell. Wie ist cine bleibende geningende Konzentration des lauswidrigen Mittels an allen Stellen des Anzuger

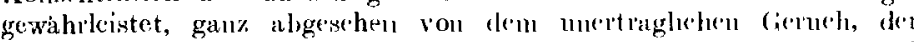

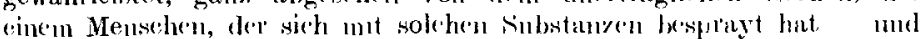

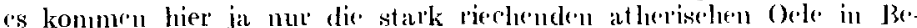

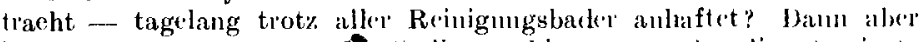

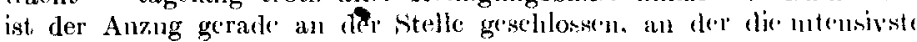
Beruhrumg nit Krankenbett 11, dgl. erfolgt. INe Preis dieses An\%ugess brträgt, nebenbei bennerkt, 18 Kront'll.

Eine andere Schutzkleidnug enpfichlt $v$. Wasielewski: Glatte Schaftstiefel oder holıe, bis \%um Knie reichende ('mum isticfel, rin bi \%ur Mitte der Wade oder dieht uber den Knochel reichender, vor'u und liuten geschlosscner', sackförmiger Mantrl ans glatt'm Gummistoff, die

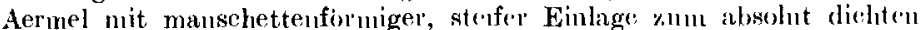
Befestigen von Gummihandsehuhe'u.

Neufeld, Elugge, Gartuer, Bujwid, Kisskalt, Uhleuhuth, Stocklosinski empfehlen Gummistoff- oder $O \cdot$.lnwhmantel nit moglielsst dichtem Aermel- und HalsabsehluB, Kopfkappe mol Gummi-

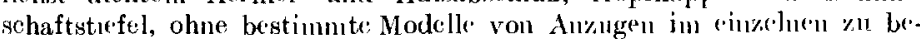
schreiben. Fligge empfiehlt reiehliehste Verwerudung von Lenkoplast

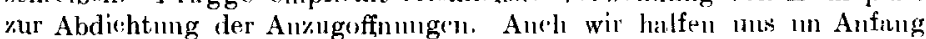
mit diesem imnerhin kostspielige'n Mittel. Dir zur Abelichtung not. wendige Leukoplastmenge kostete nach unse're' Be'pelimung pro P'reson etwa $1 \mathrm{M}$.

Auf der Quarantänestation unscres Spitals fïr die Verwuıdeten aıs den Osten kamen wir nın unabhängig von obigen Autoren zur Konstruktion von Schntzanzïgen, dic ioh Ihnen in zwei Modellen zeigen will.

Angeregt da\%u wurden wir dureh die Tatsache, dab bei Verwendung von Leinenanziigen mit (xumnistitefeln, Watte- und Lenkoplastabdichtumg sowohl Aert\%e als Wärter, die die erste Aufualıuı" der Verwuudeten unachten, regclmäßig ein paar Länse ablrokumeı. bie Gessichtspunkte, die uns loes der Herstellung des Anzuges leitelen, waten folgende:

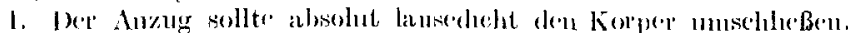

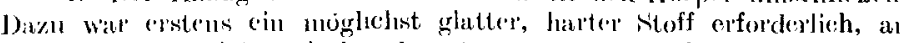

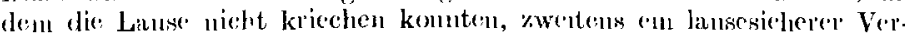
sehhul der Auzugsoffinungen.

2. Der Anzng sollte moglichst leieht und bequem seiju. Dice Lente mussen melorere fitnuden darin anshalte'n kömulen le' anstrengende. Arbeit, xum 'leil in warmen Rämmen (Sommer, Bateximmer). 'T'rots

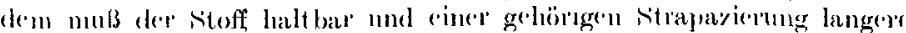
Zeit. gewatehsen sein.

Be Ntofflage wal ztemleh leicht \%u lösen. Wiehtig ist an diesen

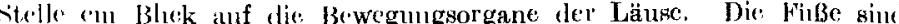
mit kleinen Hakeh'n bewaftnet, es fehlen Kaugfißße u. dgl. An glatten, harten Stoff'n komu'n steh darum Läuse nicht forthewegen, während \%. B. Fluegen mut Hilfo de' Sangfißchen daranf emporkliechen, Mucken mit Hilfe ihrer filugel dalan emporsehweben kónnen. (ef, Abb. bei Branll- teifert.) Wir wahlten flumes, geschliffenes, hellfarbiges Oeltuch, etwa von der Alt de's Blllrothbattists, nul ein engmaschigeres

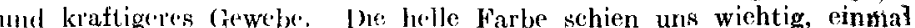
um rinen all\%u finsteren Endruek der Kleidung anf die Patienten zit vermeiden, dam aber anch, 111 beim Reingen des An\%ugs etwa, nosh clarunf befindliche Läuse leicht erkemnen zu konuen. Der Stoff ist W.1eht, da 3 das Gewicht des ge'saut 'n An\%uges nicht gan\% 3 Pfund erroicht.

Schwienig dagegene wal die Iosung des Vorschlusses. Denn hien kolliderten wir mit dem Naturgessetz, daß jedess bing anf Ere'en rine Oeffunug haben mul

Wir ver'suehten die Losung folgendermaßen: Nach Art aines

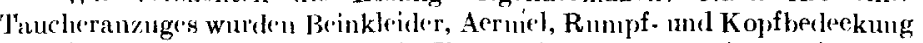

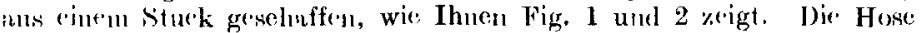

Fig. 1.

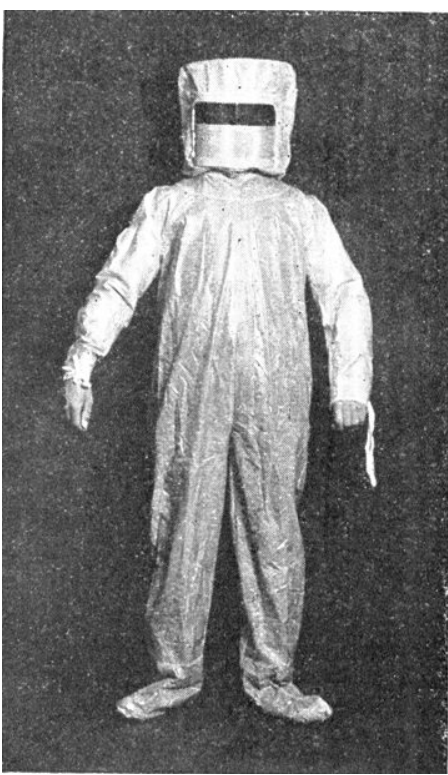

Fig. ?

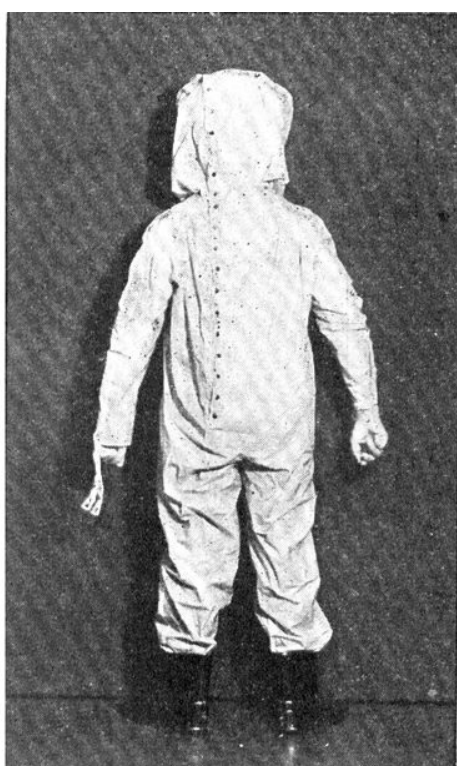

Wederkt FuB und Bein wheh Alt einer Wasserhose, die Sohle ist leicht rerstarkt durch eine doppelte Stoffschicht. Der Runpf ist durch eingelassene Falten weit und bequem gebaut, die Aermel haben einen besonder'en Versehluß. Eine an unteren Ende eingelassene Manschette chlic:Bt mittels (iummi dicht über dem Handgelenk. In den so zwischen auBerem Aermelende und Manschette entstehenden Hohlraun wird ciu länsewidriges Mittel eingefilh't, clann der außere Aerniel glatt zuGuuncugrefaltet und ein Gumnihandsehuh dariiber gezogen (Fig. 3). l) ie andere Art des Atrmelve'schlusses ist so, daß das Aermelende cine
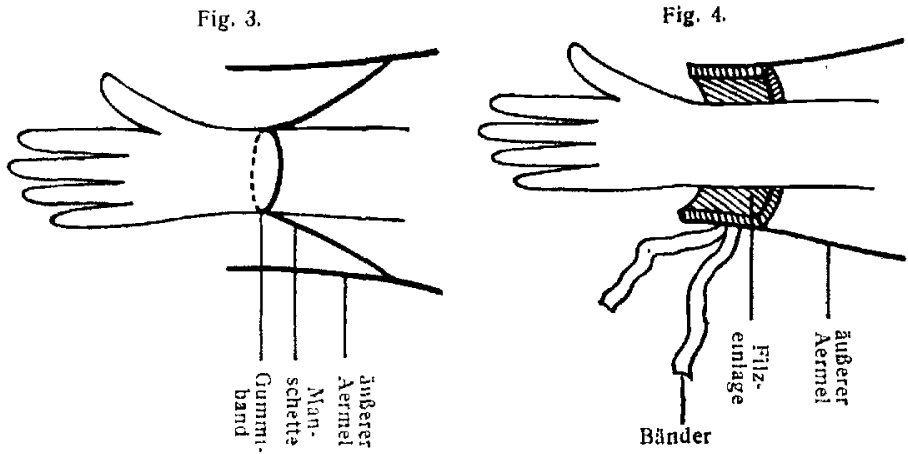
Filzeinlage besitzt, die mit lauswidriger Substanz versehen wirl, danı wird der Aerinel fest und glatt ïber dem Handgelenk zusa inmengelegt und zugebunden (Fig. 4). Dic Kopfunmiillung ist mo angelegt, daB in das Innere cine Matrosemunitze Init Gummiband eingobant ist, dadurch ist eine Verschiebung der Kopfumkleidung verhindert. Dem Gesicht entsprechend, ist eine $20 \times 30 \mathrm{~cm}$ große, in Aluninium gefaßte Maske (Tig. 5), die in ihrer oberen Hälfte aus einer Glimmerscheibe,
Fig. 5 .

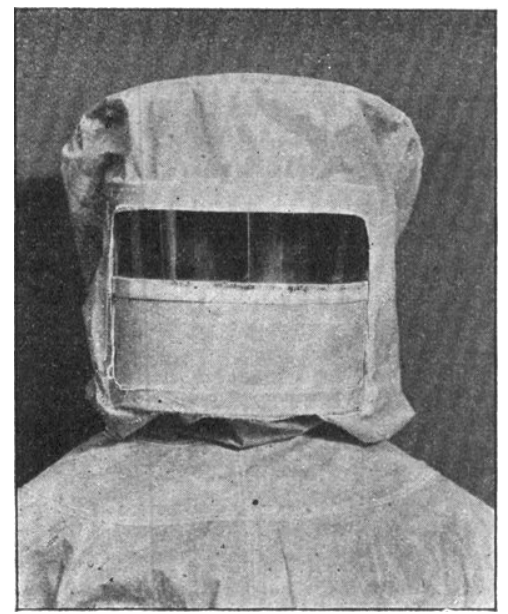
in ilure feinstmaschigem, verzinktem. also rostfreiem Drahtuetz besteht; die Größe der durehsich tigen Scheibe ermöglicht den Träger des An\%uges einen bequemen Blick nach vorn und seitlich; LGlas wurde nicht genonımen wegen der Gefahr einer möglichen Zersplitterung, Zellıloid war wegen seiner Feuergefähr]ichkeit auszuschlic:ßcn. Die Scheibc wird, $11 \mathrm{~m}$ etwaiges Beschlagen zu verhindern, vor dem Gebrauch nit Glasipur od. Lasin eingerieben.

Das Drahtnetz ist groß genug, bei einiger Uebung dem Träger eine zureichende Atmung z.u gewähren. Auch die kleinsten Läuse können dieses Drahtnetz, an dessen Rückseite eventuell noch eine düne Mull. schieht angebracht werden kann, nicht passieren. Die ganze Maske ist fest mit dem angrenzenden Stoff verklebt. Von diesen nunskeliartigen Gesichtsabsehlnß gilt natiirlich das Gleiche, wic vou allen Ro spirationsmasken. Jeder ist froh, wenn er das Ding von Gesicht wieder abnehmen kann, und es bedarf erst einer gewissen Gewöhnung an ein lïngeres Arbeiten mit solehen Masken. das sich aber ziemlich rascl erlernen läßt, zunial wenn bei Niehttragen einer solchen Maske das Schreekgespenst der Flecktyphusinfektion in Hintergrunde lauert. Ic h selbst arbeite stundenlang in diesen Anzugc ohne die geringste $\mathrm{n} B \mathrm{~B}^{-}$ schwerden dieht.

Soweit wäro also der bisher beschriebene Anzug theoretisch läuse-

Die Oeffunng des An\%uges ist an die Riekseite v:rll.gt, und der Versehluß; von der Kappe bis z.tun Rumpfende herahrieltend, so hesgestellt, wie as Ihnen der folgende (pnersehnitt elläntern soll (Fig. 6 )

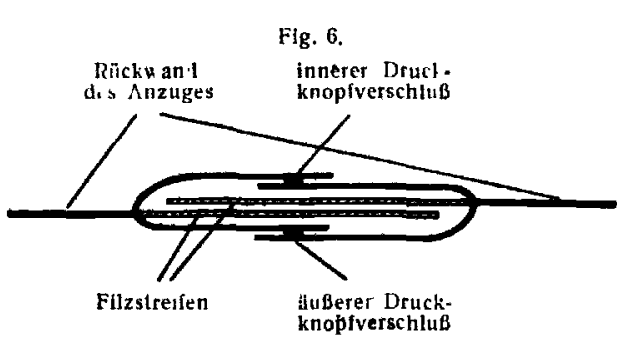
Zunächst in $\mathrm{d}(\mathrm{r}$ Tiefe ein Druckkuopfyerschluß, sturkf; dichtstehe'nct: Druckkı̈̈pfe', dann \%wei von dror gregeniberliegende Seiten ausgehende. sich iuberdeckende. "Filzstreifen, dann wieder ein oberflächlích gelegener, fester Druekknopfversehlıß. An dex Kappe und an Beinsehlit\% silul die Fil\%streifen niteinander vernaht. Dic Streifen werden vor dem Gebrauch des An\%uges nit cinem läusewidrigen Mittel ge.tränkt oder am besten mit der geruchlos $n$ grauen Salbe bestriehen. Dic Konstruktion dieses Verschlusses ist derurt, daß eine Laus, dic einzudringen versuchte, inmmer gegen einen der Filzstreifen laufen muB und durch das lausewidrige Mittel, mit dem dic Streifen versehen sind, rugrundegeht.

Die Nähte des Anzuges
Fig. 7.

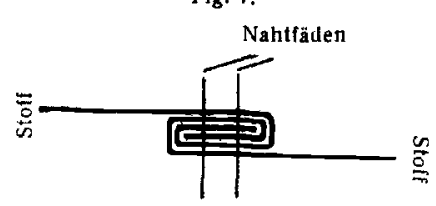
sind, wie Ihnen das nächste Bild zeigt (Fig. 7), doppelt gefalzt, also auch für eine Laus nicht passierbar.

Der Anzug wird so verwendet, daß Arzt oder Wärter, nur mit Hemd, Unterhose und Striimpfen bekleidet, in diesen hineinsteigt. Sehr zweckmäßig ist, besonders für die heißeıl Tage, eine aus dickem Flanell bestehende Hemdhose, die leicht den Schweiß aufsaugt und dann später bequem gewaschen und desinfiziert werden kann. Als Schuhe werden die üblichen Operationsstiefel benutzt. Der Anzug wird hinten nach vorheriger Präpariemung der Filzstreifen von einer zweiten Person sorgfältig geschlossen, danı die Aerınel in der oben angedeu- teten Weise mit Gummihandschuhen abgedichtet. Nach der Arboit wird der Anzug zuerst mit Kresolseifenlösung ahgewaschen und dann ansgezogen. Von Zeit zu Zeit muls der Anzug neu geölt werden.

In diesem Modell glauben wir eine absolut läuse- und überhaupt insektendichte Kleidung geschaffen zu haben.

Da aber boi diesen Anzug das, wenn aueh im Vergleich mur Gefahr der Flecktyphusiubertragung. geringe, Opfer der Unbehaglirhkeit unel vielleieht ciner vermohrten Sishweißsckrcetion gebracht werden mußs, will ich nicht versäumen, Ihnen noch ein mweites Modell \% r. reigen, das 2.war theoretisch keinen absolut länsedichten Abschluß gewährt, praktisch aber doch wohl kaum eine Lalls an den Träger des Anzuges herankonınen läßt (Fig. 8 und 9).

Hose, Rumpf-, Aermel- und Rückenverschluß sind, wie bei dem eben geschilderten Modell, anders ist nur der Kopf- und Halsschutz.
Fig. 8.

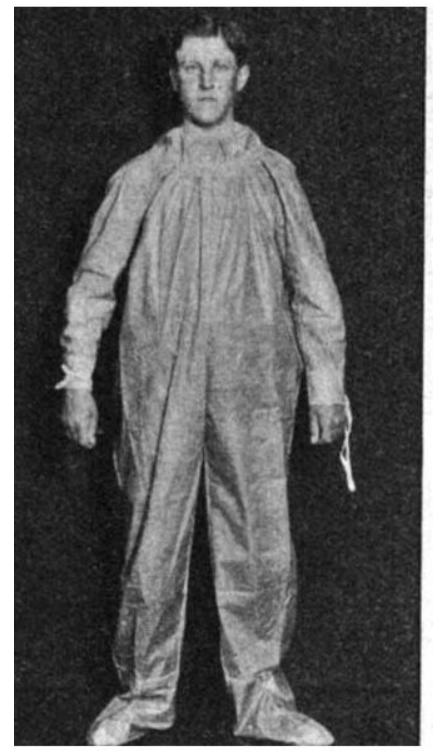

Fig. 9.

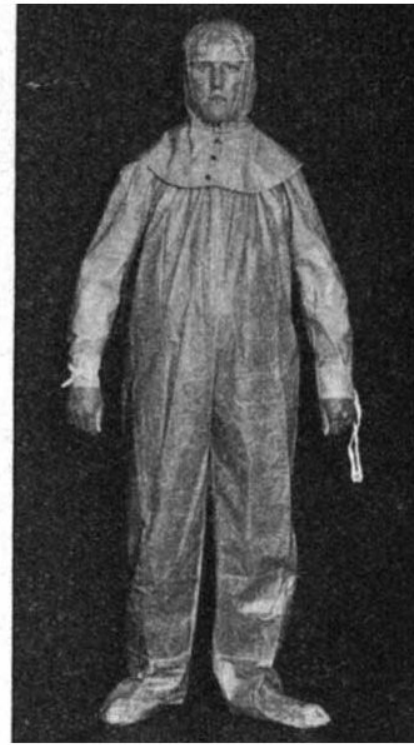

D). r rigentliche Anzung schlir.Bt moglichst dicht am Halsc ab. Durch rimen ion inmen e.ingenähten. nach auBen isberfallenden Filustreifen. der bein (acbranch nit linusewidligem Mittel verschen wird. ist erreicht. daß Länsc, die empor\%ukricehen versuchen. abgefangen umd getötet

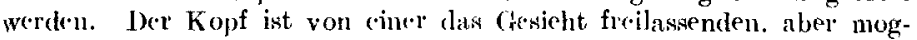
lichst clicht anliegenden Kappe beckeckt. dic inber Rioken mul Bruat weit heriberhangt. Dif. Kappe ist mit Barchent gefüttort, der am iußcren Rand: (s)enfalls nit lanswidrigem Mittel versehen werden kann. sodaß auch hier Lause, die auf der' AuBenseite 'ventuell horum. kriechen, nic den Rand der Kappe inberschreiten konnen. Der Träger d $/ \mathrm{s}$ Allzuges ist also theoretisch fur Lanse nur an dem freibleibenden desieht angreifbar. Inwieweit die cmpfindiche Gesichtshaut mit einer lauswidrigen Salbe ohme Gefahr der Entroundung cingestrichen werelen kanı. darübor fehlt ıns bisher eine genugende Erfahrung.

Fin endgültiges Urteil über die praktische Verwendbarkeit der beiden gezeigten Modelle, insbesondere, ob sie in Wirklichkeit einen läusesicheren Abschluß gewähren, muß erst durch längere Beobachtung, in diesen Falle im ,Menschenexperiment", gewonmen werden. Der Krieg wird uns ja wohl leider reichlich Zeit und Material dazu zur Verfügung stellen.

Die Anzuge wurden vou der Firna stein metz \& HehI. Hamburg, Schaartor. hergestellt. der wir volerst die alleinige Fabrikation inbertragen haben. Der Preis des einzelnen Anzunges stellt siol je; nach Größe auf $25-30 \mathrm{Nr}$. 